\title{
Interacción madre-hijo durante la alimentación en niños con hipotiroidismo congénito
}

\author{
Rolando Rivera González,* Miriam Figueroa Olea,* Citlali Soto Ramos, ** \\ Fabiola Soto Villaseñor, ** Carmen Sánchez****
}

RESUMEN

Introducción: La interacción madre-hijo es un elemento esencial para el desarrollo integral del niño, especialmente durante los primeros años de vida y en presencia de enfermedades como el hipotiroidismo congénito o condiciones socioemocionales del cuidador y la familia. Objetivo: Evaluar la interacción madre-hijo durante la alimentación en díadas con niños con hipotiroidismo congénito e intervención temprana, y compararla respecto a díadas con niños sanos. Material y métodos: Estudio observacional, comparativo, transversal y prospectivo. Se formaron tres grupos: 1) díadas con niños con hipotiroidismo congénito e intervención temprana bajo tratamiento hormonal sustitutivo; 2) díadas con niños sanos sin intervención temprana; 3 ) díadas con niños sanos ( $\sin$ hipotiroidismo) con intervención temprana. La edad de los niños fue de 0 a 12 meses. Se visitaron los hogares para videograbar la interacción durante la alimentación del niño. Se comparó la interacción entre los tres grupos con las puntuaciones totales y por subescalas de la Nursing Caregiver Assessment Feeding Scales. Resultados: El Grupo 1 obtuvo calificaciones más altas en la interacción, seguido por el Grupo 3 y con menores puntajes el Grupo 2. Conclusiones: La intervención temprana favorece la interacción madre-hijo. Las diferencias esperadas en la interacción por la condición socioeconómica o la escolaridad materna fueron menores ante la exposición a la intervención temprana.

Palabras clave: Interacción madre-hijo, intervención temprana, hipotiroidismo congénito.

\section{Mother-infant interaction during feeding time in children with congenital hypothyroidism}

\begin{abstract}
Introduction: Mother-infant interaction is essential for the overall infant development, especially during the first years of life, and in the presence of diseases such as congenital hypothyroidism or socio-emotional adverse conditions of the caregiver or the family. Objective: To evaluate the mother-infant interaction during feeding sessions in dyads with children with congenital hypothyroidism included in an early intervention program and compare dyads with healthy children. Materials and methods: Observational, comparative, cross-sectional and prospective study. Three groups were formed: 1) 159 dyads, children with congenital hypothyroidism who received early hormone replacement therapy and early intervention program; 2) 254 dyads with healthy children without early intervention program, and 3) 59 dyads with healthy children (no hypothyroidism) with early intervention program. The age of the children was 0-12 months. Dyads home were visited
\end{abstract}

* Laboratorio de Seguimiento del Neurodesarrollo. Instituto Nacional de Pediatría;

** Maestría en Rehabilitación Neurológica. Universidad Autónoma Metropolitana-Xochimilco.

Recibido para publicación: 02/05/2014. Aceptado: 19/05/2014.

Correspondencia: Citlali Soto Ramos. Universidad Autónoma Metropolitana, Prolongación Canal de Miramontes 3855, Col. Ex-Hacienda San Juan de Dios, Delegación Tlalpan, C.P. 14387, México, D.F. E-mail: sotocitlalienf08i@hotmail.com

Este artículo puede ser consultado en versión completa en http://www.medigraphic.com/enfermerianeurologica 
to videotape the interaction during feeding moment of the child. Interaction between three groups with total scores and subscales of Nursing Caregiver Assessment Feeding Scales was compared. Results: Group 1 scored higher on interaction, followed by Group 3 and Group 2, with lower scores. Birth order and maternal age had greater association with scores on the total test $(\mathrm{p}<0.01)$. Conclusions: Major differences in the children's scores were observed. Early intervention program strategies promote mother-infant interaction. The expected differences in interaction related with socioeconomic status or maternal education were lower with the effect of the exposure to an early intervention program.

Key words: Mother-child interaction, early intervention, congenital hypothyroidism.

\section{ANTECEDENTES}

$\mathrm{L}$ a interacción entre el niño y su madre u otros cuidadores es reconocida como un factor promotor y de bienestar para el desarrollo infantili,' la importancia de las interacciones tempranas para el funcionamiento socio-emocional posterior del niño ${ }^{2}$ y las formas de interacción que se establecen entre los niños y sus padres repercuten en el desarrollo temprano del lactante ${ }^{3,4}$ y en crecimiento socioemocional de los niños de 12 a 24 meses. ${ }^{5}$

Barnard (1976) y sus colegas de la Facultad de Enfermería de la Universidad de Washington generaron un modelo de evaluación de la interacción durante la alimentación al que denominaron Nursing Caregiver Assessment Feeding Scales (NCAFS), ${ }^{6}$ que describe la interacción madre-hijo como un diálogo recíproco donde cada uno de los participantes tiene responsabilidad para mantener fluyendo el proceso interactivo, destacando respuestas contingentes del uno al otro (vocalizaciones, contacto visual y físico, gesticulaciones faciales, lenguaje corporal, afecto, estimulación de respuestas cognitivas y motrices) y patrones comunicativos (pausas maternas, toma de turnos, la claridad de las señales y la sensibilidad y responsabilidad a las mismas). ${ }^{6}$ Se han realizado investigaciones con la NCAFS, asociándola con el desarrollo, como indicador de impacto de programas de intervención o como resultante de condiciones socioemocionales específicas. Morisset demostró la importancia de la interacción madre-hijo como predictor del desarrollo cognitivo y del lenguaje; ${ }^{7}$ otros autores han estudiado la interacción madre-hijo a través de la calidad de las respuestas maternas a las señales del niño y su relación con el desarrollo infantil;; ${ }^{2,8}$ Barnard (1996) estudió la relación entre la intervención temprana, la responsibidad del cuidador hacia las necesidades del niño y cómo ambas favorecen su desarrollo. ${ }^{9}$

Las interacciones no sólo deben ubicarse en un contexto biológico sino además, se debe considerar la complejidad del contexto de las relaciones sociales en las cuales se realiza la interacción; ${ }^{10,11}$ Evans y colaboradores ${ }^{2}$ describen a la pobreza como generador de estrés materno y que las madres en esa condición disminuyen la responsibidad hacia su hijo, debilitando su capacidad para atender las necesidades tanto básicas como favorecedoras de un óptimo desarrollo.

El hipotiroidismo congénito (HC) se puede definir como el evento resultante de la disminución de la actividad biológica de las hormonas tiroideas a nivel tisular, ya sea por producción deficiente o bien, por resistencia a su acción en los tejidos diana. ${ }^{12} \mathrm{El} \mathrm{HC}$ es la primera causa de retardo mental prevenible, lo cual es posible sólo si se detecta a tiempo, pues permite un tratamiento temprano.

Pese a un inicio de tratamiento precoz, se han reportado en la literatura diversas alteraciones en el desarrollo de estos niños, como trastornos en la percepción visuoespacial, psicomotricidad y lenguaje, así como en la atención y memoria. El inicio tardío del tratamiento o la severidad del hipotiroidismo determinan que los niños con ese padecimiento se consideren aún como una población de riesgo para alteraciones en el desarrollo, a lo que se suman condiciones psicosociales adversas, frecuentes en la población de bajos recursos, éstas son contrarrestadas incorporándolos en un programa de intervención temprana (IT) para favorecer su desarrollo, cuyas principales características son facilitar las interacciones tempranas, la exposición a experiencias de aprendizaje, relaciones de juego y exploración del medio. Dicho programa enfatiza las funciones del cuidador primario, que generalmente es la madre, para favorecer las expectativas de desarrollo posterior del niño en las acciones cotidianas de su cuidado y crianza.

El objetivo del presente trabajo fue evaluar la interacción madre-hijo durante la alimentación en díadas con niños que tuvieron diagnóstico de $\mathrm{HC}$ y participaron en un programa de intervención temprana (IT), comparándola respecto a díadas con niños sanos con y sin (IT).

\section{MATERIAL Y MÉTODOS}

La población objetivo fueron díadas con niños diagnosticados con HC que acudieron al Instituto Nacional de Pediatría (INP) para su manejo y control hormonal sustitutivo y al programa de intervención temprana (IT) del Laboratorio de Seguimiento del Neurodesarrollo de ese instituto entre los años 2003 y 2010. El programa es un 
modelo orientado a promover el desarrollo integral del niño durante los primeros años de vida, así como las competencias de cuidado y crianza organizadoras del desarrollo; ${ }^{13}$ una de sus bases es favorecer las interacciones tempranas del niño con su entorno. También se incluyeron otros dos grupos de comparación: un segundo grupo formado por díadas con niños sin HC y sin IT (sinHC/sinIT) y un tercer grupo formado por díadas con niños sin $\mathrm{HC}$ pero que sí recibieron IT (sinHC/conIT).

Se explicaron los procedimientos a realizar y se solicitó consentimiento informado, incluyendo la visita domiciliaria en un horario regular de alimentación del niño para la videograbación de la interacción; se solicitó a la madre que la realizara como lo hace de manera cotidiana, según los criterios expuestos en el manual de la NCAFS $^{14}$ y el entrenamiento de certificación del mismo para su aplicación.

La NCAFS es una prueba utilizada para valorar la interacción madre-hijo durante la alimentación, consta de 76 reactivos que se califican en una escala binaria (observado o no observado), los reactivos se encuentran divididos en seis subescalas. Los primeros 50 reactivos se dividen en las cuatro primeras subescalas, y califican a la madre en relación con la actitud hacia el niño (sensibilidad a las señales, respuesta a la angustia, fomento al crecimiento socioemocional y fomento al crecimiento cognitivo); los 26 reactivos restantes califican al niño en relación con la actitud hacia el cuidador y se distribuyen en dos subescalas (claridad de las señales y responsibidad al cuidador). De los 76 reactivos, 18 se consideran contingentes (15 provienen de las subescalas del cuidador y tres del niño), consistentes en comportamientos interactivos sincrónicos entre los miembros de la díada, en los cuales no basta que la interacción sea propuesta por uno de ellos, sino que, además, debe obtenerse la respuesta correspondiente del otro. ${ }^{14}$

\section{RESULTADOS}

Se evaluó la interacción madre-niño en 472 díadas, divididas en los tres grupos descritos en la metodología. No se observaron diferencias entre los grupos por edad, escolaridad, estado civil o condición socioeconómica de las madres; en el grupo con HC predominó el sexo femenino (es propio de la epidemiología del padecimiento).

Las puntuaciones alcanzadas en los totales y subescalas de la NCAFS en cada uno de los grupos se presentan en el cuadro I. Las puntuaciones fueron mayores en el Grupo 1 respecto a los otros dos grupos $(\mathrm{p}<0.001)$; fueron significativamente más altas que las del Grupo 2 en todos los totales y puntuaciones por subescalas. Respecto a las diferencias entre los Grupos 1 y 3 , ambos con IT, se observan mejores puntuaciones de manera global en los niños con HC/IT, pero la diferencia sólo fue estadísticamente significativa entre estos dos grupos en las siete puntuaciones indicadas con las letras "a" y "c" de la columna de significancias del cuadro I. Las puntuaciones del grupo 3 fueron superiores a las del Grupo 2 en 9 de las 12 puntuaciones estimadas indicadas con las letras "a" y "b" de la columna de significancia del cuadro I, excepto "Total del niño", "Fomento al Crecimiento Cognitivo" y "Claridad de las Señales".

Al ser la población de niños con $\mathrm{HC}$ de especial interés en nuestro estudio, se analizaron diferencias en los resultados de la NCAFS según las características del HC, no encontrándose diferencias por los niveles hormonales al inicio del tratamiento, tipo de hipotiroidismo, ni por edad de inicio del tratamiento.

Se analizó la relación entre las variables generales de la madre con la puntuación total de la NCAFS; se observó (Cuadro II) una tendencia que reflejó que entre mayor escolaridad, mejores puntuaciones en la NCAFS; sin embar-

Cuadro I. Promedios totales y por subescalas del Nursing Caregiver Assessment Feeding Scales en los tres grupos.

\begin{tabular}{|c|c|c|c|c|c|}
\hline $\begin{array}{l}\text { Grupo } \\
\text { Puntuación NCAFS }\end{array}$ & $\begin{array}{c}\text { 1. } \mathrm{conHC/conIT} \\
\text { (159) }\end{array}$ & $\begin{array}{l}\text { 2. } \sin H C / \sin I T \\
(254)\end{array}$ & $\begin{array}{c}\text { 3. } \sin H C / \text { conlT } \\
\text { (59) }\end{array}$ & $\begin{array}{l}\text { Total } \\
(472)\end{array}$ & $P^{\&}$ \\
\hline Total & $59.6 \pm 12.4$ & $47.1 \pm 10.6$ & $52.3 \pm 10.6$ & $52.0 \pm 12.6$ & $\mathrm{a}$ \\
\hline Total madre & $39.0 \pm 8.4$ & $31.9 \pm 7.4$ & $36.3 \pm 6.9$ & $34.9 \pm 8.3$ & $\mathrm{~b}$ \\
\hline Total niño & $20.6 \pm 5.2$ & $15.1 \pm 4.0$ & $16.0 \pm 4.4$ & $17.1 \pm 5.2$ & $\mathrm{c}$ \\
\hline Total contingencia & $13.2 \pm 4.2$ & $9.6 \pm 3.7$ & $12.1 \pm 3.7$ & $11.1 \pm 4.2$ & $\mathrm{~b}$ \\
\hline Total contingencia madre & $11.0 \pm 3.5$ & $8.5 \pm 3.2$ & $10.3 \pm 2.9$ & $9.6 \pm 3.5$ & $\mathrm{~b}$ \\
\hline Total contingencia niño & $2.2 \pm 1.0$ & $1.1 \pm 0.9$ & $1.8 \pm 1.0$ & $1.6 \pm 1.0$ & $\mathrm{a}$ \\
\hline 1. Sensibilidad a las señales & $12.8 \pm 2.7$ & $9.8 \pm 2.6$ & $11.4 \pm 2.1$ & $11.0 \pm 2.9$ & $\mathrm{a}$ \\
\hline 2. Respuesta a la angustia & $9.2 \pm 1.6$ & $8.6 \pm 1.7$ & $9.4 \pm 1.5$ & $8.9 \pm 1.7$ & $\mathrm{~b}$ \\
\hline 3. Fomento socioemocional & $11.1 \pm 2.7$ & $8.9 \pm 2.6$ & $10.1 \pm 2.4$ & $9.8 \pm 2.8$ & $\mathrm{a}$ \\
\hline 4. Fomento cognitivo & $6.0 \pm 2.5$ & $4.6 \pm 2.3$ & $5.4 \pm 2.5$ & $5.2 \pm 2.5$ & $d$ \\
\hline 5. Claridad de las señales & $12.5 \pm 2.8$ & $9.9 \pm 2.2$ & $9.5 \pm 2.5$ & $10.7 \pm 2.8$ & $\mathrm{c}$ \\
\hline 6. Responsabilidad al cuidador & $8.1 \pm 2.7$ & $5.2 \pm 2.3$ & $6.5 \pm 2.2$ & $6.4 \pm 2.7$ & a \\
\hline
\end{tabular}

\& $p$ de $\mathrm{F}$ en la diferencia de medias, ANOVA $p<0.001$, prueba post hoc de Tukey compara los tres grupos a: $1 \neq 2 \neq 3$, b: 1 y $3 \neq 2, \mathrm{c}: 1 \neq 2$ y 3 , d: $1 \neq 2$ 
Cuadro II. Promedios de puntaje total del Nursing Caregiver Assessment Feeding Scales en los tres grupos, según las variables de la madre.

\begin{tabular}{|c|c|c|c|c|c|c|c|c|}
\hline Grupo & $\begin{array}{l}\text { 1. conHC/conlT } \\
\text { (159) }\end{array}$ & $P^{\&}$ & $\begin{array}{l}\text { 2. } \sin H C / \sin I T \\
(254)\end{array}$ & $P^{\&}$ & 3. $\operatorname{sinHC/con~IT~}$ & $P^{\&}$ & $\begin{array}{l}\text { Total } \\
(472)\end{array}$ & $P^{\&}$ \\
\hline \multicolumn{9}{|l|}{ Escolaridad } \\
\hline Analfabeta/incompleta & $57.6 \pm 11.8$ & & $41.8 \pm 11.2$ & & $45.0 \pm 7.5$ & & $47.9 \pm 13.4$ & \\
\hline Primaria & $59.0 \pm 12.6$ & & $47.2 \pm 10.5$ & & $54.3 \pm 13.8$ & & $53.4 \pm 12.9$ & \\
\hline Secundaria & $59.1 \pm 12.9$ & 0.4235 & $46.9 \pm 10.1$ & 0.1106 & $52.0 \pm 11.6$ & 0.8173 & $51.0 \pm 12.2$ & 0.0899 \\
\hline Preparatoria & $62.9 \pm 11.2$ & & $48.62 \pm 11.2$ & & $52.9 \pm 9.5$ & & $53.9 \pm 12.6$ & \\
\hline Universidad & $61.0 \pm 14.9$ & & $49.7 \pm 9.8$ & & $56.5 \pm 0.7$ & & $53.0 \pm 11.2$ & \\
\hline \multicolumn{9}{|l|}{ Estado civil } \\
\hline Casada & $63.7 \pm 10.0$ & & $48.2 \pm 9.9$ & & $50.86 \pm 9.0$ & & $52.8 \pm 11.9$ & \\
\hline Unión libre & $57.2 \pm 13.2$ & $0.0020^{*}$ & $46.4 \pm 11.2$ & $0.0112^{*}$ & $51.71 \pm 11.0$ & 0.3780 & $51.6 \pm 13.0$ & 0.1354 \\
\hline Soltera/sep. & $54.0 \pm 11.8$ & & $40.6 \pm 10.6$ & & $55.92 \pm 12.3$ & & $48.6 \pm 13.3$ & \\
\hline \multicolumn{9}{|l|}{ Nivel socioeconómico } \\
\hline Medio bajo & $58.8 \pm 12$ & & $48.5 \pm 9.7$ & & $58.3 \pm 6.0$ & & $50.8 \pm 10.9$ & \\
\hline Bajo & $60.0 \pm 13$ & 0.5891 & $44.6 \pm 11.1$ & $0.0026^{*}$ & $52.0 \pm 10.7$ & 0.3196 & $53.4 \pm 13.5$ & $0.0010^{*}$ \\
\hline Muy bajo & & & $39.6 \pm 15.0$ & & & & $39.6 \pm 15.0$ & \\
\hline
\end{tabular}

\& $p$ de $\mathrm{F}$ en el análisis univariado para la diferencia de medias. ${ }^{*} p<0.05$

go, las diferencias no son estadísticamente significativas utilizando la comparación de medias. Adicionalmente, se estimó esta relación mediante análisis de correlación, observándose correlaciones significativas sólo en el Grupo 2. Respecto a la edad materna, no se encontraron relaciones importantes, pero sí con el estado conyugal de la madre: las madres solteras presentan puntuaciones significativamente más bajas en los dos primeros grupos respecto a las casadas ( $p>0.05)$; no se observa esa relación en el Grupo 3. Respecto a la ocupación de la madre, no se encontraron diferencias estadísticas; respecto a la condición socioeconómica, se observaron sólo diferencias en el Grupo 2, que no recibió estimulación, en el que a mejor condición socioeconómica, mayores puntuaciones en la NCAFS.

\section{DISCUSIÓN}

La interacción cuidador-niño se ha considerado como un organizador del desarrollo infantil en edades tempranas. Adicionalmente, la presencia de un padecimiento como el HC puede afectar las formas de relacionamiento de la madre con el niño, siendo ello un factor de riesgo para presentar alteraciones en el desarrollo ${ }^{15} \mathrm{o}$ afectar la implementación eficiente de las estrategias impartidas desde el programa de IT. Se eligió la prueba NCAFS por ser una prueba con la que se han realizado múltiples reportes en la literatura internacional y muestra adecuada capacidad de diferenciar condiciones de riesgo que están descritas como vinculadas con la interacción temprana.

Los resultados de los niños con HC se comportan cercanos a los estándares de otros reportes y al manual de la NCAFS (61.30 puntos), ${ }^{14}$ y por encima de los grupos de referencia que se utilizaron como controles, siendo las díadas sinHC/sinIT las que obtuvieron la puntuación más baja (47.1) y las díadas con HC/IT quienes obtuvieron la puntuación más alta (59.6). Debe señalarse que el grupo de referencia descrito en el manual de la NCAFS es tres años mayor al de las madres de los niños de nuestro estudio.

Las diferencias en la interacción entre el grupo HC/IT respecto al grupo sin $\mathrm{HC}$ ni IT son mayores en el segundo semestre de vida, en el que los niños tienen mayor exposición al programa. Pensamos que las orientaciones que se proporcionan en un programa con las características descritas favorecen la capacidad del cuidador de mostrarse más responsivo a las demandas del niño, interpretar las señales que emite, incorporar estrategias de juego y aprendizaje en las acciones y contener la ansiedad del niño cuando ésta se produce. La capacidad de los programas de estimulación, intervención o atención temprana para impactar en la interacción ha sido descrita como variable de interés en algunos trabajos..$^{916-18}$

El que las correlaciones entre la escolaridad de la madre y la puntuación en la NCAST sólo fueran significativas en el grupo sin IT (Grupo 2), indica que el tener un mayor grado de educación influye positivamente en la interacción madre-hijo, ${ }^{19,20}$ pero esta correlación disminuye cuando la díada se expone a un programa de IT; la escala en la que se observó que la escolaridad influyó positivamente fue en la de sensibilidad a las señales. ${ }^{21}$

Respecto a la edad de la madre, observamos que -al igual que en otros reportes- las madres adolescentes del grupo sin IT califican más bajo respecto a las madres adultas. Sin embargo, esa tendencia no se observó cuando las madres asisten al programa de IT; ello puede interpretarse 
nuevamente como un beneficio de la IT sobre las madres adolescentes y las redes de apoyo que se propician cuando se integran al programa, además de las estrategias de interacción.

Finalmente, el nivel socioeconómico de la familia se relacionó de manera global con la interacción; tomando los tres grupos juntos, se observó menor puntuación general en el nivel socioeconómico más bajo; sin embargo, los Grupos 1 y 3, que recibieron IT, no mostraron diferencias significativas, lo cual interpretamos como un beneficio de la intervención de reducir diferencias derivadas de la condición socioeconómica, ya que en el Grupo 2 (sin IT), se presentaron diferencias cercanas a los nueve puntos entre las condiciones socioeconómicas muy baja y medio baja.

Como se refirió anteriormente, la capacidad del programa de IT de impactar positivamente en la interacción depende de un tiempo de exposición; sin embargo, existen casos en los que el efecto del programa sobre la interacción puede ser mayor en algunas díadas que otras, lo que nos lleva a preguntar sobre otras condiciones que faciliten o limiten el efecto de la IT sobre la interacción. En nuestras díadas, la interacción fue menor en el grupo con baja escolaridad, madres solteras y el nivel socioeconómico muy bajo. Sin embargo, se observó que el programa benefició más la interacción precisamente en las díadas con baja escolaridad y de condición socioeconómica muy baja; ello puede reflejar que las carencias de información y formas de interactuar en las madres en estas condiciones obtienen mayores beneficios de la asistencia al programa, logrando diferenciarse positivamente de sus similares que no asisten..$^{19,20}$

Los mejores resultados en los niños con IT indican que el programa tiene un importante impacto. Este tipo de programas provee elementos esenciales que facilitan los estilos de crianza e interacción de la madre, ${ }^{22}$ lo cual se podría observar cualitativamente si se hiciera un análisis de los reactivos en los que se presentaron las mayores diferencias por la exposición a la IT, y que pueden verse como fortalezas logradas a partir de la inserción en el programa -aspecto que no se incluye en el presente reporte pero que consideramos necesario integrar en futuras comunicaciones-.

Los hallazgos encontrados en nuestra investigación expresan principalmente que la inclusión de díadas a un programa de IT favorece la interacción y contribuye con el desarrollo del niño, incorporándola como estrategia promotora en tales programas para reforzar las competencias maternas en la crianza.

Propiciar una buena interacción madre-hijo desde los primeros momentos ${ }^{23}$ dará pauta para que el desarrollo del niño sea integral, además de forjarse evolutivamente desde las primeras etapas del ciclo vital para, posteriormente, poder relacionarse con sus semejantes, su medio y sus intereses, contribuyendo a constituirse como un ser íntegro en una sociedad en la cual pueda desenvolverse plenamente.

\section{BIBLIOGRAFÍA}

1. Fonagy P. Attachment, the development of the self, and its pathology in personality disorders: PSYCHOMEDIA Telematic Review 1997 [citado octubre de 2005]. Available from: http://www.psychomedia.it/ $\mathrm{pm} /$ modther/probpsiter/fonagy 2. htm.

2. Evans G, Boxhill L, Pinkava M. Poverty and maternal resposiveness: The role of maternal stress and social resources. Int J Behav Dev. 2008; 32: 232-237.

3. Maldonado J, Feintuch M. Salud mental del bebé. Intervenciones durante el embarazo. Perinatol Reprod Hum. 2001; 15 (1): 31-41.

4. Lartigue T. Relación materno fetal en México: aspectos transculturales. Perinatol Reprod Hum. 2001; 15 (1): 75-88.

5. Hodges EA, Houck GM, Kindermann T. Reliability of the nursing child assessment feeding scale during toddlerhood. Issues Compr Pediat Nur. 2007; 30: 109-130.

6. Barnard K. NCAST II learner's resource manual. In: Sumner GSA, editor. Seattle: NCAST Publications; 1976. p. 8.

7. Morisset CE. It takes two to communicate: The role of interpersonal experience in child language acquisition. Seattle: University of Washington; 1988.

8. Mc L, Snyder L. A transaccional approach to early language training. Columbus: Merriel; 1978.

9. Barnard K, Osofsky J, Beckwith L, Hammond M, Appelbaum M. A collaborative effort to study mother-child interaction in three risk groups: Social risk mother, adolescent mother, preterm infant. Infant Ment Health J. 1996; 17 (4): 293-301.

10. Juarez MC. Influencia cultural en el vínculo madre-infante. México: UPN; 2004.

11. Cravioto J, Arrieta R. Nutrición, desarrollo mental, conducta y aprendizaje. 2a ed. México: Sistema Nacional para el Desarrollo Integral de la Familia, Instituto Nacional de Ciencias y Tecnologías; 1985.

12. Mayayo E. Hipotiroidismo y bocio. Asociación Española de Pediatría [Internet]. 2011; 77: 1: [150-65 pp.]. Available from: http://www.aeped. es/sites/default/files/documentos/13_hipotiroidismo_y_bocio.pdf.

13. Rivera GR, Sánchez C, Figueroa OM. Vigilancia y promoción del desarrollo infantil. México, DF: Textos Mexicanos; 2009.

14. Sumner G, Spietz A. NCAST caregiver/parent-child interaction feeding manual. Seattle: NCAST Publications, School of Nursing, University of Washington; 2004.

15. Figueroa OM. Interacción madre-hijo en niños con hipotiroidismo congénito menores de un año de edad que reciben el programa de intervención temprana. Rev Cienc Clin. 2004; 5 (2): 57-67.

16. Badr LK, Garg M, Kamath M. Interaction for infants with brain injury: result of a randomized controlled study. Infant Behav Dev. 2006; 29 (1): $80-90$.

17. Han KJ, Kwon M. Effects of a mother-fetus interaction promotion program on the mother-infant interaction during feeding. J Korean Acad Child Health Nurs. 2007; 13 (2): 191-200.

18. Brady JE. Los oscuros secretos de los mayas: la exploración arqueológica de las cuevas. In: Grube $\mathrm{N}$ et al, editors. Mayas, una civilización milenaria. China: Könemann; 2006. pp. 297-307.

19. Leigh D, Helen E, Heather M. Mother-infant interaction in premature infants at three months after nursery discharge. Int J Nurs Pract. 2003; 9 (6): 374-381. 
20. Brandt KA, Andrews CM, Kvale J. Mother-infant interaction and breastfeeding outcome 6 weeks after birth. J Obst Gynecologic Neonatal Nurs. 1998; 27 (2): 169-174

21. Cho K. A study on the mother-infant interaction in teaching and feeding situations. Taehan Kanho. 1990; 29 (5): 54-68.
22. Muñoz-Ledo P, Sánchez C, Méndez I, Mandujano M. Sistemas diádicos (madre-hijo) y secuela al año de edad en niños con daño neurológico de origen perinatal. Perinatol Reprod Hum. 2003; 17 (1): 10-19.

23. Bryan A. Enhancing parent-child interaction with a prenatal couple intervention. MCN Am J Matern Child Nurs. 2002; 25 (3): 139-145. 